\title{
Web Based 3D Visualization and Interaction for Whole Body Laser Scans
}

\author{
Sandy RESSLER*, Kyle LEBER \\ National Institute of Standards and Technology, USA \\ http://dx.doi.org/10.15221/13.166
}

\begin{abstract}
A web based system that allows the user to select, sort, and measure 3D bodies using displays inside of ordinary web browsers without the use of 3D plug-ins is presented. Our system, AnthroWeb3DMeasure (AW3DM), allows the user to visually explore a set of 3D laser scanned bodies. The bodies we use come from the CAESAR[3] data set, however nothing inherently limits applicability of these techniques to this specific data set.

The user is presented with a set of controls, the 3D view of the body, and a tabular view of demographic data associated with each body. We use X3DOM[1] as the basis for the 3D body visualizations. X3DOM is a "declarative 3D" language that enables us to place the graphical elements of the body directly into the DOM (Document Object Model) of the web page itself. This has significant implications for the extensibility and practical usefulness of the system as it is not dependent on arcane plugins and is interoperable among all modern web browsers.
\end{abstract}

Keywords: Anthropometry, Web3D, X3D, WebGL, web browser, visualization

\section{Introduction}

We present a web based system for the display and measurement of anthropometric data using modern web browsers. The AnthroWeb3DMeasure (AW3DM) system, illustrated in Figure 1, also demonstrates the use of a hybrid approach to graphics that utilizes "declarative 3D" graphics to allow the use of web infrastructure tools combined with binary geometry to improve performance.

Along with the display of the body surface the anthropometric landmarks are also highlighted with bright spheres directly on the body. These spheres may be selected and, when clicked, information about the landmark appears in a dialog box. This system builds on previous work [2] with the significant difference of working within the context of a non-modified web browser, and no longer requiring specialized plug-in's or separate applications.

Controls arranged in an accordion interface allow the user a variety of manipulations. One can a) view the model from viewpoints constructed to view individual landmarks and their surrounding areas, b) scale up individual landmarks, and c) highlight body segments. In addition there is a "virtual measurement" system. The user can click on any portion of the body surface, not simply a landmark, and obtain $x, y, z$ position information in units ( $\mathrm{mm}$ or inches) that correspond to the original data of the model. We are currently working to extend the measurement capabilities to planar (2D) and volumetric (3D) measures.

Embedding the 3D visualization directly into the web browser opens up the rich variety of web development tools for use in anthropometry applications. In addition the use 3D visualization and measurement techniques built right into the capabilities of the web browser enables widespread diffusion of this technology both for educational and research applications. Web browsers are ubiquitous and we no longer have to battle the significant interoperability problems of previous plugins and specialized applications. This system functions in any modern browser and has been demonstrated to function on all major computing platforms (Macintosh, MS Windows PCs and Linux).

*sressler@nist.gov

\footnotetext{
${ }^{1}$ DISCLAIMER: Any mention of commercial products is for information only; it does not imply recommendation or endorsement by NIST.
} 


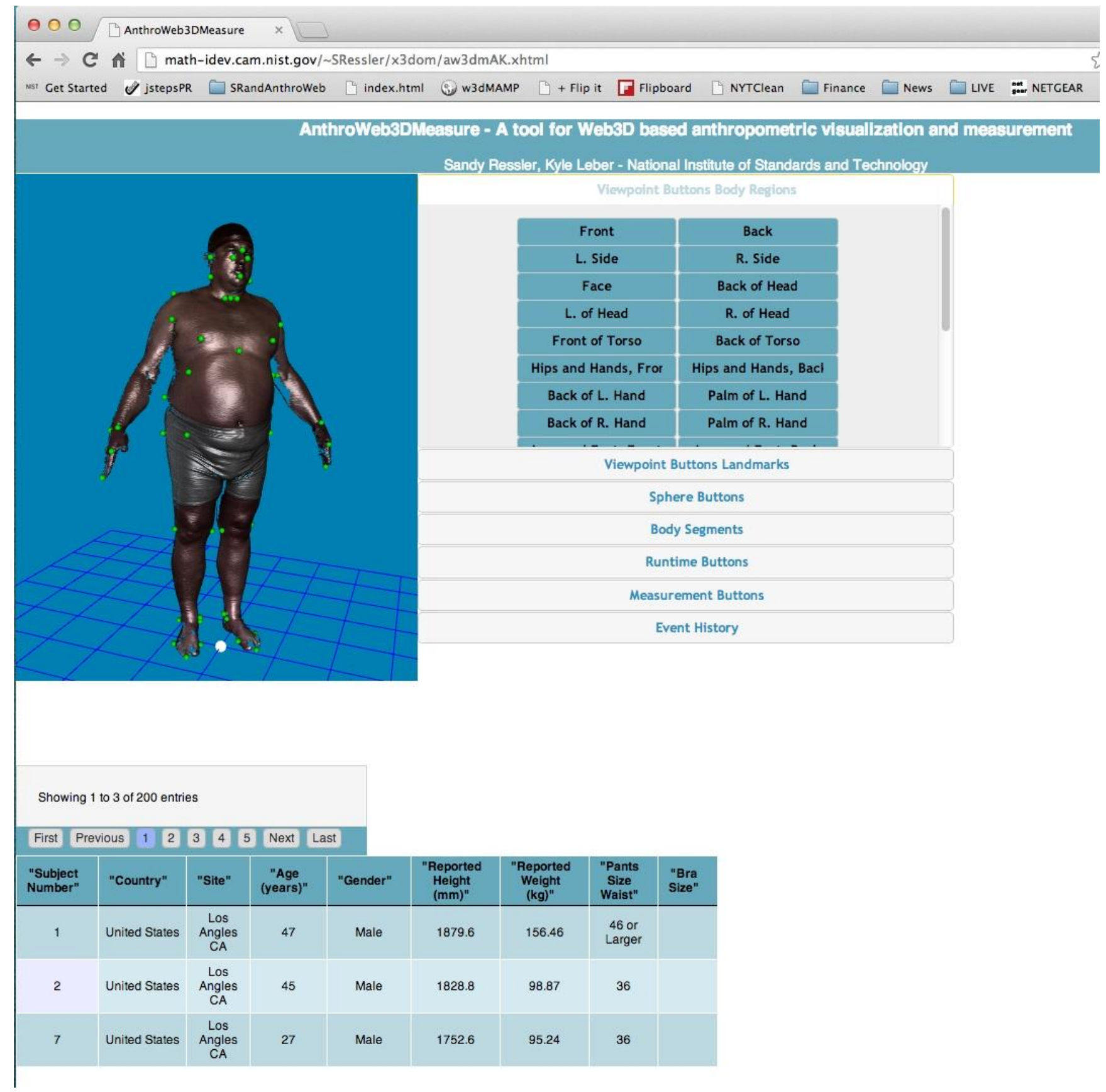

Figure 1: AnthroWeb3DMeasure application in use.

In Figure 1, note that the layout and design of the application takes advantage of typical web UI constructs. Next to the 3D body display is an accordion type interface, and in the open panel, a set of buttons. Below the figure is a tabular display of data along with associated user interface controls, all of which are made available via the inclusion of a table JavaScript library, that works seamlessly in the web page. The significance of all of these types of interfaces and development methodology is to demonstrate the ability to integrate a complex 3D application in the context of a web page without the use of exotic plug-ins or software. Collected together this is simply a web page that happens to have some 3D model of a whole body laser scan and the manipulation of the application uses commonly understood web controls. 


\section{Data Processing}

Before we can do any measurements or manipulation we must first obtain the data: the actual 3D laser scan of the body. In this case we use scans from the CAESAR [3] project. The original scans exist as .ply files for the 3D geometry of the body and ascii txt files containing the $x, y, z$ positions of the landmarks. We process the ply files into series of $x 3 d$ files. In the original version of this system the x3d files were place into the HTML pages, but these pages quickly become unwieldy to edit. The current version of the system refers to the $x 3 \mathrm{~d}$ files via the $\mathrm{x} 3 \mathrm{~d}$ command "INLINE" which references the external files. This is similar to the $C$ programming \#include directive.

The processing from ply to $\mathrm{x} 3 \mathrm{~d}$ is done via the AOPT utility, provided by Fraunhoffer as part of their instantreality suite of tools[4]. AOPT offers many types of geometry compression and conversion utilities. We compress the geometry which greatly improves the user experience and performance. Simply put placing thousands of polygons into the DOM does not work. The performance of the web browser becomes unusable. This hybrid approach of compressing the geometry while still retaining the declarative nature of most of the items in the scene is quite effective.

We followed instructions similar to the examples in the AOPT documentation [5]:

...an example is shown how to also accomplish mesh optimization (here of a ply model) by calling aopt three times, for cleanup, mesh patching (for coping with the 16 bit indices limit), and final binary geometry creation.

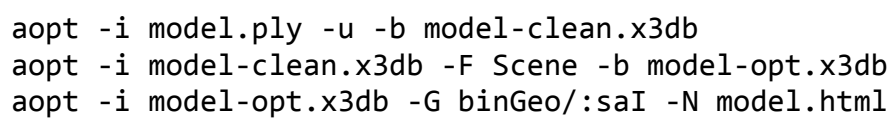

Currently 200 subjects are included in the system.

\section{Web3D Technologies}

Our Web-based solution is made possible by the convergence of a number of relatively new technologies. The two key technologies are WebGL and X3DOM. X3D[6] is an ISO standard representation for graphics and some interactivity. X3DOM is an effort to place X3D graphics inside of the DOM of a web page, and render the graphics directly in the page. This is accomplished via a JavaScript library acting as a "polyfill". The term polyfill has become the defacto description of a capability that could, or should, be supported natively in a web browser, but isn't just yet. The X3DOM JavaScript library enables web browsers to display X3D graphics without the installation of plugins.

X3DOM functions much better when the graphics are rendered using WebGL. (Notably however, you can use other systems to render the graphics such as Flash for those browsers that do not support WebGL.) WebGL is simply an implementation of OpenGLES inside of the browser. It add significant performance improvements to the display of graphics inside of a web page, as the rendering is now able to take advantage of the graphics processing hardware. Simply put, real-time "game-like" graphics are now possible inside of web pages.

\subsection{System Architecture/Leveraging Web Development Infrastructure}

Developing a web-based 3D application has advantages, and disadvantages. First the downside: performance is clearly an issue as the web browser is an asynchronous environment and updates don't necessarily take place in the order you expect and performance is generally more sluggish than a standalone dedicated application. The big upside, however, to web-based development is the robust and amazingly active developer community and sheer number of supporting libraries and support knowledge out on the web. As a concrete example we utilize the jQuery[8] library which has become a ubiquitous support library easing JavaScript programming.

\subsection{Declarative 3D}

One of the most interesting aspects of this system is the use of X3DOM and it's "declarative" nature. Exactly, what does this mean? First some terms, declarative versus imperative. A graphics system such as OpenGL is imperative. Operations are defined and executed right away painting on the screen as fast as possible, you tell the computer how to do an operation. Declarative graphics tells the computer what to do, not how to accomplish the task. 
Declarative markup is one of the foundational technologies that enabled the World Wide Web to exist. HTML, the HyperText Markup Language, is also a declarative markup language. The markup for a portion of the page that is a paragraph " $<p>$ " simply identifies that the associated text is a paragraph, and says nothing about how to render the paragraph. The Cascading Style Sheet (CSS) style sheet language associates the structurally identified elements with how to render them. This means that a $<p>$ in one page can look totally different depending on the styles of the page. The classic "CSS Zen Garden" [7] web site helped define and solidify this capability.

The "big win" using declarative graphics is the ability to leverage web infrastructure for your graphics application. In this case the anthropometric landmarks are visually represented via spheres. We can select all of the spheres via their "class", a convenient method to group all landmark spheres for action. The simple jQuery statement $\$$ ('.l landmarkSphere') will select all of the landmark spheres in the scene, and we can perform arbitrary actions on them such as color or scale changes.

It becomes trivial to add arbitrary semantic information to the geometry. Figure 2, for example, demonstrates the ability to group landmarks according to body segments such as the "left arm". We simply define a ".leftarm" class and associate that class with the appropriate landmarks. Next we apply some visual indicator to that class producing the desired visual results. This is of course a contrived example, however we could just as easily drive the visuals from more meaningful data, such as the location of diseases or injuries. The infrastructure provided by this declarative approach supports the visual display of any number data driven applications.

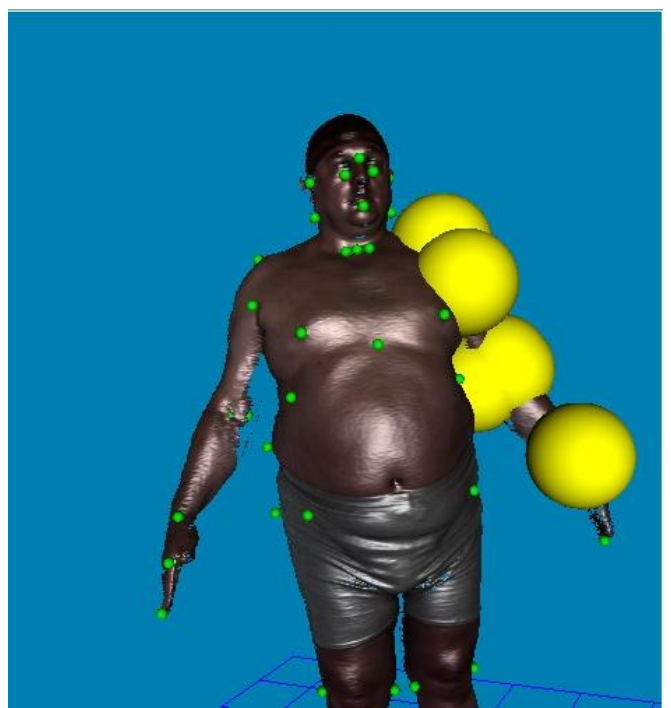

Figure 2. Arbitrary semantics associated with landmarks.

\section{Body Specific Capabilities}

\subsection{Data Tables}

The tables in the lower portion of the screen contain the demographic information for each subject. In addition, clicking on the subject number brings that subject into view in the main display portion of the screen. Each numeric column of data is sortable by clicking on the header, making it trivial to display the largest or smallest subject, by weight, height or other column. The functionality of the data table also illustrates the advantage of doing web-based development. The table is a jQuery library, freely available and simple to add into the application. It has been robustly tested and has a lot of typical tabular abilities (such as the sorting) that we can incorporate. 


\begin{tabular}{|c|c|c|c|c|c|c|c|c|c|c|c|}
\hline \multirow{2}{*}{\multicolumn{6}{|c|}{ Showing 1 to 10 of 200 entries }} & \multicolumn{6}{|c|}{ Showing 1 to 10 of 200 entries } \\
\hline & & & & & & & & \multirow{3}{*}{$\begin{array}{l}\text { First - Pre } \\
\text { "Reported } \\
\text { Height } \\
(\mathrm{mm}) "\end{array}$} & $5-\sqrt{-2}$ & $-\sqrt{4}-\sqrt{5}$ & Next - Last \\
\hline \multirow{3}{*}{$\begin{array}{l}\text { "Subject } \\
\text { Number" }\end{array}$} & \multirow{3}{*}{ "Race" } & \multirow{3}{*}{$\begin{array}{l}\text { First - Pr } \\
\text { "Reported } \\
\text { Height } \\
(\mathrm{mm})^{4}\end{array}$} & ous $-1-2$ & $\sqrt{4} \sqrt{5}$ & Next - Last & \multirow{2}{*}{$\begin{array}{l}\text { "Subject } \\
\text { Number" }\end{array}$} & \multirow[b]{2}{*}{ "Race" } & & \multirow{2}{*}{$\begin{array}{l}\text { "Reported } \\
\text { weight } \\
\text { (kg)" }\end{array}$} & \multirow{2}{*}{$\begin{array}{c}\text { "Pants } \\
\text { Size } \\
\text { Waist" }\end{array}$} & \multirow{2}{*}{$\begin{array}{l}\text { "Bra } \\
\text { Size") }\end{array}$} \\
\hline & & & \multirow{2}{*}{$\begin{array}{l}\text { "Reported } \\
\text { Weight } \\
(\mathrm{kg}) "\end{array}$} & \multirow{2}{*}{$\begin{array}{c}\text { "Pants } \\
\text { Sizze } \\
\text { Waist" }\end{array}$} & \multirow{2}{*}{$\begin{array}{l}\text { "Bra } \\
\text { Size") }\end{array}$} & & & & & & \\
\hline & & & & & & \multirow{2}{*}{72} & \multirow{2}{*}{$\begin{array}{c}\text { Other Not } \\
\text { Listed } \\
\text { Above }\end{array}$} & \multirow{2}{*}{1625.6} & \multirow{2}{*}{51.25} & & \multirow{2}{*}{$\begin{array}{c}\text { No } \\
\text { Response }\end{array}$} \\
\hline 1 & White & 1879.6 & 156.46 & $\begin{array}{l}46 \text { or } \\
\text { Larger }\end{array}$ & & & & & & & \\
\hline 2 & White & 1828.8 & 98.87 & 36 & & 216 & White & 1625.6 & 58.96 & & $\begin{array}{l}\text { Dont } \\
\text { Know }\end{array}$ \\
\hline 7 & $\begin{array}{l}\text { Spanish/Hispanic } \\
\text { Mexican American }\end{array}$ & 1752.6 & 95.24 & 36 & & 17 & White & 1574.8 & 99.77 & & $42 c$ \\
\hline 8 & White & 1828.8 & 77.10 & 33 & & 236 & White & 1625.6 & 65.76 & & $40 \mathrm{~d}$ \\
\hline 9 & White & 1778 & 89.80 & 34 & & 84 & White & 1778 & 77.10 & & $40 c$ \\
\hline 10 & African American & 1828.8 & 102.04 & 38 & & 113 & White & 1828.8 & 136.05 & & $40 c$ \\
\hline 12 & White & 1714.5 & 76.19 & 34 & & 213 & $\begin{array}{c}\text { African } \\
\text { American }\end{array}$ & 1625.6 & 81.63 & & $38 d$ \\
\hline 13 & White & 1701.8 & 65.76 & 30 & & & Atrican & 1498.6 & 52.15 & & \\
\hline 14 & White & 1574.8 & 63.49 & & $34 \mathrm{~b}$ & 36 & American & 1498.6 & & & \\
\hline \multirow[t]{2}{*}{15} & White & 1574.8 & 56.69 & & 32b & 86 & White & 1625.6 & 88.44 & & $38 \mathrm{c}$ \\
\hline & & & & & & 87 & White & 1689.1 & 68.93 & & $38 \mathrm{c}$ \\
\hline
\end{tabular}

Figure 3. Data tables for left and right images, with pagination controls on top.

\subsection{Anthropometric Landmark Information}

The anthropometric landmarks come from a separate file, distinct from the geometric shape of the body. This file contains the $x, y, z$ positions for the 73 landmarks measured for the CAESAR study data. As Figure 4 shows, clicking on the green sphere, one of the landmarks, causes a pop-up display to appear giving the name of the landmark.

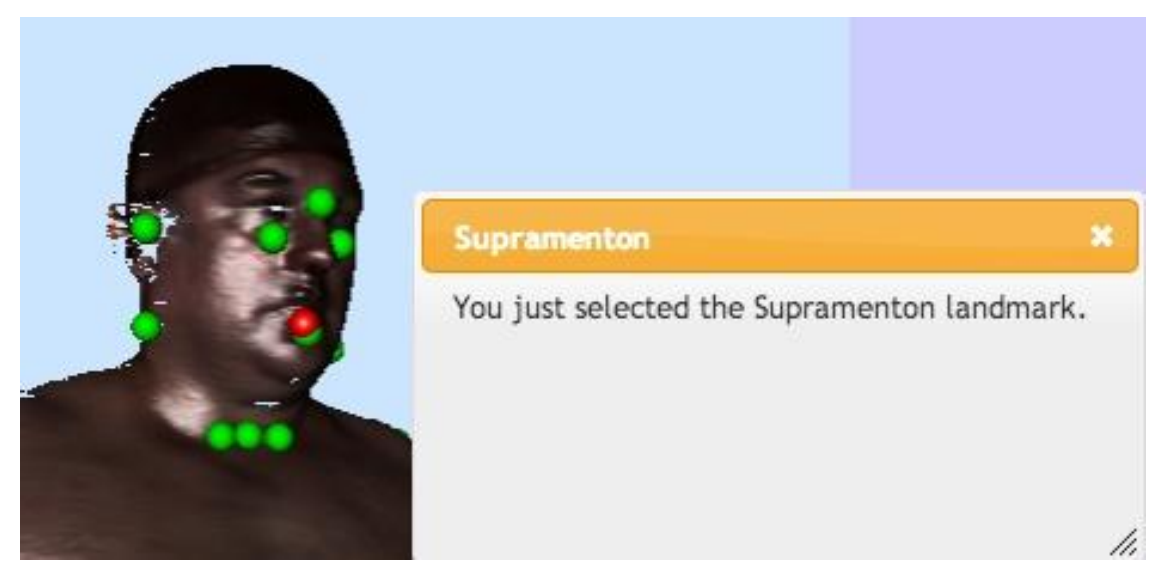

Figure 4. Anthropometric landmark selected with information displayed.

\subsection{Measurements}

Figure 5 illustrates clicking on any part of the body will result in a measurement displayed in the dialog. This is the measurement of a single point. You can also bring up a measurement dialog and measure along a polyline, continuously clicking along the body and getting measurements, between the points. The measurements are along a straight line and in this implementation do not follow the curvature of the body. Which we leave for future work.

In Figure 6, virtual yardsticks can appear in the corner of the display volume. Units can be in millimeters, centimeters or inches. 


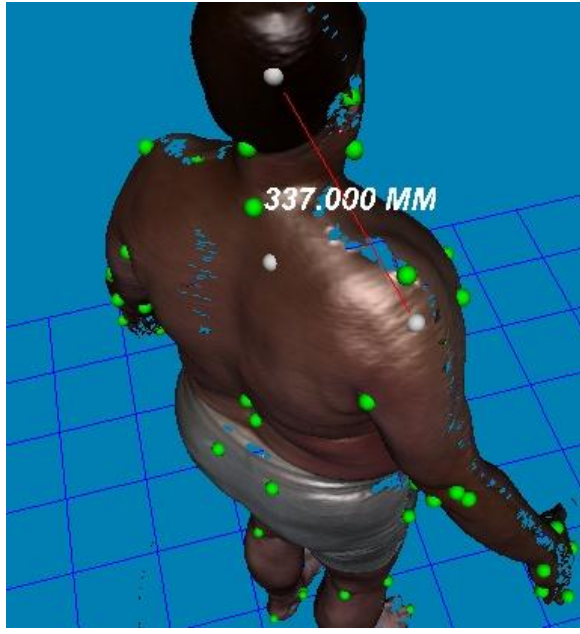

Figure 5. Distance Measurement.

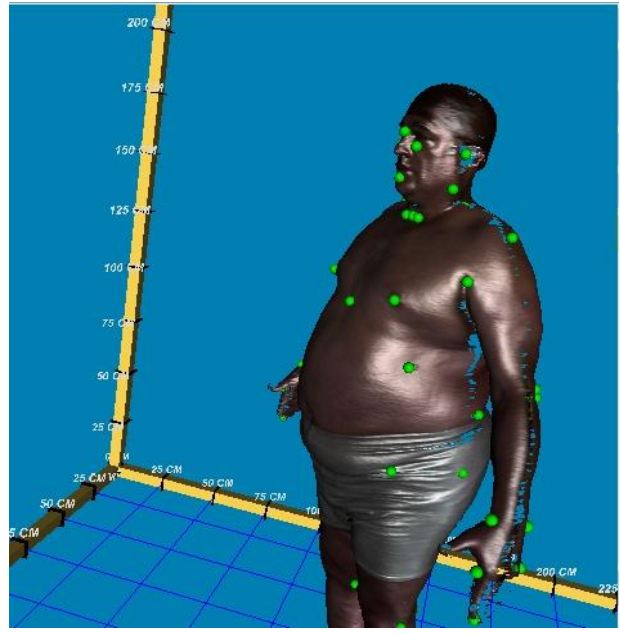

Figure 6. "Yardstick" style rulers in corner of body display area.

Comparisons between two bodies can be performed in a convenient manner. When the system is configured for dual body display the X3D Model Comparison dialog, illustrated in Figure 7 can be used. It allows the user to select two landmarks on each model and see the distances between the landmarks.
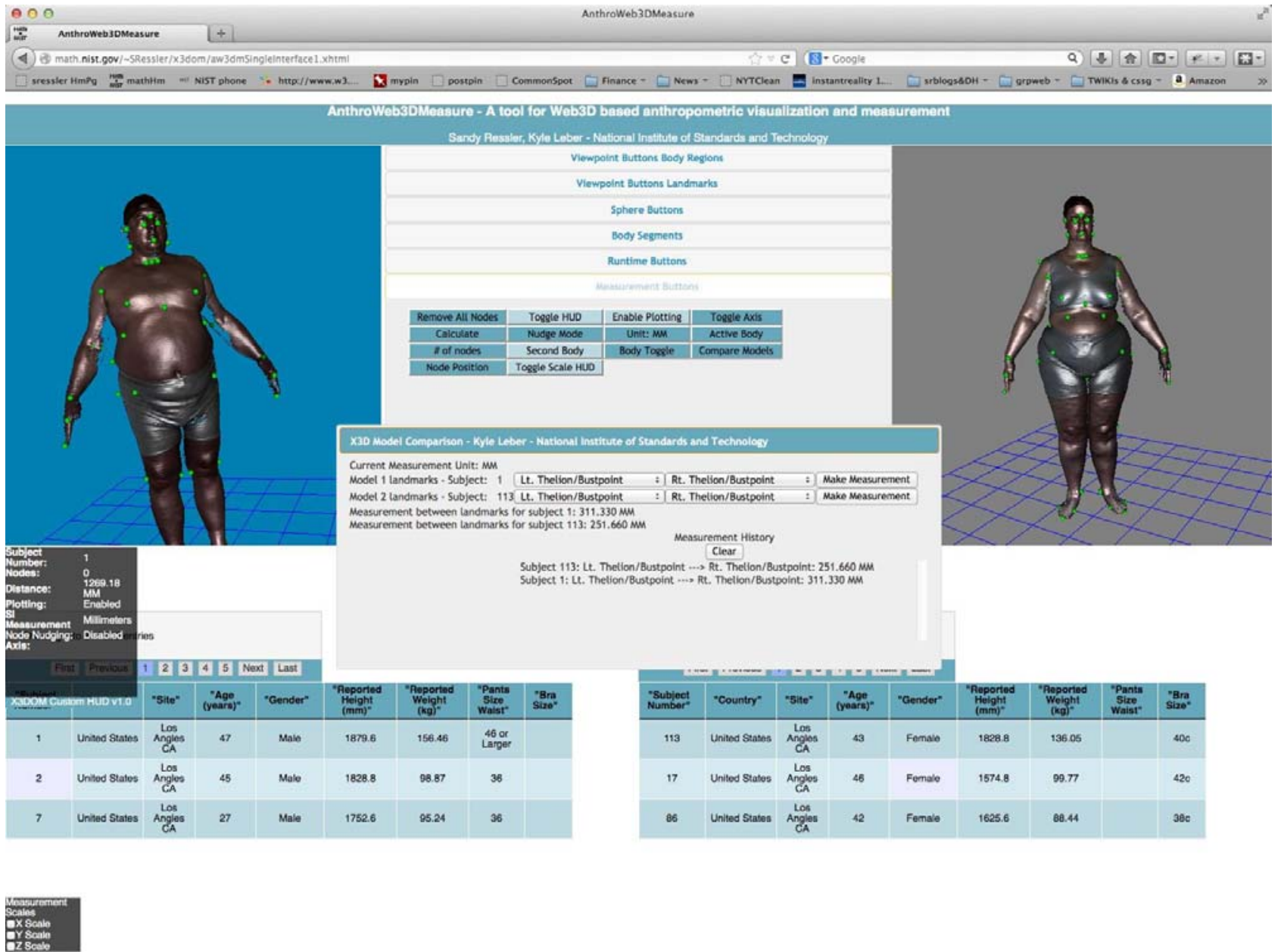

Figure 7. Model comparison dialog with dual body display. 


\section{Conclusions and Future Work}

Out system provides the rudimentary tools needed to perform anthropometric measurements using only a modern web browser. Special software, installation procedures, or licensing arrangements are not needed in order to perform sophisticated measurements. Most importantly we would like to be able to import, visualize and analyze a variety of data sets. Currently the only data set we have used is the CAESAR survey. Datasets which we could analyze should be interchangeable allowing comparisons between the measurements taken from one survey to another (where the measurements are semantically meaningful to compare). Finally the source code to this system will be made freely available and we intend on following normal "open source" conventions such as placing the code in a Github repository for further development.

\section{References}

1. Behr, J., Eschler, P., Jung, Y., AND Zollner “ , M. 2009. (2009): “X3DOM - a DOM-based HTML5/ X3D integration model”, In Proc. Web3D 2009, ACM Press, New York, USA, 127-135.

2. Ressler, Sandy, (2001): "A Web-based 3D Glossary for Anthropometric Landmarks", in Proceedings of HCl International 2001, New Orleans, LA.

3. Robinette, K., Blackwell, S., Daanen, H., Boehmer, M., Fleming, S., S., Brill, T., Hoeferlin, D., and Burnsides, D., (2002): "Civilian American and European Surface Anthropometry Resource (CAESAR), Final Report, Volume I: Summary, AFRL-HE-WP-TR-2002-0169”, Air Force Research Laboratory, Human Effectiveness Directorate, Crew System Interface Division, 2255 H Street, Wright-Patterson AFB OH 45433-7022 and Society of Automotive Engineers International, 400 Commonwealth Drive, Warrendale PA, 15096

4. Fraunhoffer "instantreality" (accessed 2013) http://www.instantreality.org/downloads/

5. Analyzing and optimizing your model for the 3D Web (accessed 2013) http://x3dom.org/docs/dev/tutorial/aopt.html

6. X3D and Related Specifications (accessed 2013) http://www.web3d.org/x3d/specifications/

7. CSS Zen Garden, (accessed 2013) http://www.csszengarden.com/

8. Resig, J. (2009). Jquery-javascript library. http://jquery.com/ 